\title{
Module Stabilizing of Biocarbon Based Electrochemical Capacitor
}

\author{
Nirwan Syarif ${ }^{1,2,3^{*}}$, Dedi Rohendi ${ }^{1,2,3}$, Wulandari Sudarsono ${ }^{4}$, Wai Yin Wong ${ }^{4}$ \\ ${ }^{1}$ Department of Chemistry, Universitas Sriwijaya, Indonesia \\ ${ }^{2}$ Center of Research Excellent in Fuel Cell and Hydrogen, Universitas Sriwijaya, Indonesia \\ ${ }^{3}$ National Center for Sustainable Transportation Technology, Institut Teknologi Bandung, Indonesia \\ ${ }^{4}$ Universiti Kebangsaan Malaysia, Institut Sel Fuel, Malaysia \\ *Email: nsyarif@unsri.ac.id
}

\begin{abstract}
One of the disadvantages of the electrochemical capacitor (EC) or supercapacitor compared with batteries is its low specific energy. It limits of EC to meet the energy needs of the electrical-electronic devices, such as electric cars. To overcome those limitations, it needs a serial circuit to increase the voltage range, and parallel circuits to increase the storage capacity. Practically, the module that built from 2-6 pieces of 2.5V EC cells will not feasible to make the module with the voltage of 5-15 V. It was found that the voltage of the EC cell could decreases to about $2.0 \mathrm{~V}$, so that the capacitance of the module significantly reduced. This paper reports the basic methods that can be applied to overcome these problems by using a stabilizing or balancing component. The balancing components used in this study were a resistor, a Zener diode, and a Schottky diode. Each component was attached to every EC cell. The influence of the Zener and Schottky diode was observed as a component of a blocking diode. The results showed that the use of a 100-ohm resistor and Zener diode reduces voltage peaks while the use of blocking diode modules leads to increased discharge time. In general, there was no significant change in the charging time, both with and without the balancing and blocking component.
\end{abstract}

\section{Keywords}

Carbon; Charging; Discharging; Galvanostatic; Stabilization; Supercapacitor

\section{Introduction}

Biomass-based carbon materials have gained considerable attention in the last few years due to their high chemical and thermal stability, tunable pore size distribution, and high specific surface areas. These properties made biomass-based carbon become a primary choice in supporting electrochemical storage, battery, and electrochemical capacitor (EC) manufacturer [1]. Electrochemical storage or electrochemical conversion is one of the ways to develop green technology in generating electricity and reducing $\mathrm{CO}_{2}$ emissions due to its high efficiency and the ease of $\mathrm{CO}_{2}$ sequestration [2]. The other advantages of biomass-based carbon are its low-price and high-availability, making it the perfect substitute for polymer-based carbon electrodes in EC [3]. The use of carbon in EC is very limited because of their distinct crystallography and morphologies, or allotropies [4].

EC, which also known as a supercapacitor, generally has very low operation voltage, i.e., 2.5-3.0 V [5]. However, gadgets, tools, cars, machines, and other electrical devices require a higher voltage than that individual cell. Cars, for instance, need $12 \mathrm{~V}$ or $42 \mathrm{~V}$ to functionalize their electrical parts. By using simple mathematics, the required voltage is supplied by 5 to 20 EC cells in series. In order to operate in electrical devices, EC should be connected in series and parallel configurations to form a module in order to meet the value in the performance parameter [6].

Almost all of the ECs produced in the laboratory or factory, are not exactly the same. Hence, the seriesconnected EC stacks develop unequal voltage distributions [7]-[9]. It became worse due to the variation of device capacitance, and the difference between DC bias voltages. Voltage distribution in a series stack of EC is initially a function of capacitance. After the stack has been held at a certain voltage for a period of time, a voltage distribution becomes a function of internal parallel resistance (leakage current). For example, consider a stack of 20 capacitors initially charged to $50 \mathrm{~V}$, if the cells have identical capacitance, the voltage should divide evenly so that each EC charges up to $2.5 \mathrm{~V}$. If the cells have any variation in capacitance, individual cell voltages will vary based on capacitance. The cells with greater capacitance should be charged to lower voltages, and the cells with smaller capacitance should be charged to higher voltages. This is due to each cell conducts the same charging current, and the voltage is a function of current and capacitance. 
This paper reports the methods that can be used to overcome these problems by using stabilizing or balancing components. The balancing components used in this study were a resistor, Zener diode, and Schottky diode. Each component was attached to every single cell of EC. The influence of the Zener and Schottky diode was observed as a component of a blocking diode.

\section{Literature Review}

\subsection{The role of voltage stabilization in energy storage}

Electric current with sufficient power and energy is required to operate the engine [10]. The operation process is short-lived by the significant voltage drop. Voltage drop phenomenon is caused by the impedance or resistance in the storage device [11]. This can cause a malfunction of other equipment connected to the power source (storage device) [12]. Furthermore, the discharging process that occurs in the storage device takes place at different speeds, and it affects the service life of the device. Therefore, voltage stabilization during the charging and discharging process in the device is required. A charged storage device, such as a battery and a supercapacitor have its own ability to stabilize the voltage [13].

In the battery, the process of storing and releasing large amounts of energy take plenty of time [14]. This causes difficulties in providing the short-term high power needed for voltage stabilization. In addition, the battery power is slowly charged, and, in some applications, the life time is very short. Temperature also influences the discharge stabilization. These stabilization factors limit energy storage applications [15].

EC fills the gaps between classical capacitors used in electronics and general batteries, because of their nearly unlimited cycle-stability as well as extremely high-power capability and their many orders of magnitude higher energy storage capability. This storage device still exhibits a large development potential that could lead to much greater capacitance and energy density than conventional capacitors, thus enabling compact designs.

ECs can be an auxiliary power source to complement the main energy source. They have a high specific power and low specific energy [16]. The two main features are the extremely high capacitance values, of the order of thousands farads, and the possibility of very fast charges and discharges due to extraordinarily low inner resistance, which are features not available in conventional batteries [17]. ECs have high durability, high reliability, no maintenance, long lifetime and operation over a wide temperature range and in diverse environments (hot, cold, and moist) [18] with efficiency is typically around $90 \%$. Their electrodes have longer lifetimes without any degradation. However, the solvent used in the capacitors remains as a shortcoming, since it decomposes after treated more than 1.23 volt [19]. Their waste can be easily recycled or neutralized, so that they are environmental friendly. ECs are not suitable for the storage of energy over longer period of time, because self-discharging rate is high, their energy density is low.

In an electric energy storage system, most fluctuations are very short (less than one second). ECs can be employed to handle such situation because of their quick charge and discharge feature. Their high specific power features suit to power high specific energy device. The application range of ECs can be increased by combining them with other forms of energy storage (e.g. batteries) to create hybrid energy storage systems that offer both high power capacity and good energy storage capacity [13]. Considerations in designing storage systems with ECs are the low working voltage of a single capacitor cell (lower than 3 V).

\subsection{Requirements for voltage stabilization}

Ideally, voltage stabilization in energy storage, especially in the vehicle must have these characteristics: high power density, high efficiency, discharge duration at its rated power (or higher if possible) for a minimum of some minutes, high reliability, very fast response time, flexible chargingdischarging rate and minimum efficiency change with the rate of discharge. In the other hand, electric vehicle manufacturers around the world are trying to increase fuel savings. One of the increasingly popular approaches is the use of on-off switching on the engine. This switch turns off the internal combustion engine when the vehicle has slowed down, and turned it back on when the driver's feet are removed from the brake. This process can reduce harmful exhaust emissions so that it reduces $\mathrm{CO}_{2}$ emissions.

Currently, the best-performing systems are using a starter/alternator driven by the belt to restart the engine. A fast engine start requires a very high current from the onboard battery, which is about a hundred amperes. As a result, even though the energy in the battery is still quite high and the cable impedance is low, the voltage drop in the circuit is still quite high. Voltage stabilization system on electric or hybrid 
vehicle power supplies using EC is carried out by Ayad et al. [20]. The system is a simple addition to the existing power network. The system is installed between the negative terminals of the energy storage device and can be installed on the vehicle chassis base.

Full electric vehicles use no fuel at all, instead of using battery - EC or battery - fuel cell hybrid systems. The $\mathrm{EC}$ unit is connected directly to the DC-link, while the battery is connected through the DC/DC converter. In this configuration, the current of the battery can be fully controlled by the converter in any driving condition in order to protect the battery from the bursts of charging/discharging currents. In the other words, DC/DC converters work to match voltage levels and regulate power flows in the system [21]. The most common bi-directional DC/DC converter uses diode in its circuit and resistor as well. Hybrid system of EC battery in the energy storage of electric vehicle makes dynamics in energy - power performances and allows the battery of less power to be installed. In the other hand, EC allows the energy from and to the battery runs smoothly, thus improve operating conditions of full electric vehicles. EC can supply higher currents and provide a larger amount of power for a considerable period of time compared to conventional capacitors but shorter time compared to batteries

\section{Materials and Methods}

For the purpose of simplification, the study of 3-6 ECs in series were employed. We proposed periodical charge-discharge cycle in the following settings: constant current, I, was $4 \mathrm{~A}$, both for charging and discharging process. The potential between two polarities was to reach an overvoltage or maximum value of 9-12 V on the EC module and $2.5 \mathrm{~V}$ on each cell. Digital power supply equipped with ampere meter and voltmeter (H\&K 1502DD, Taiwan) was used as the energy supply. The power supply also has capability to automatically adjust the current that flow through the module.

The data acquisition board (Labjack, U3-LV, USA) which equipped with multi-channel monitoring program in personal computer was used to observe the change in voltage that occur both in the module and the individual cells. The program was developed by using Labjack library for Labview graphical language (National Instrument, USA) [22]. A blocking component for the module is Zener and Schottky diode deployed in series to the module. On the other hand, the balancing component was connected in parallel to each cell [23]. It was assumed that the voltage of each cell is similar to one another. The balancing component used is a diode or resistor. Figure 1 shows the scheme of blocking and balancing component usage in the EC cells and module.

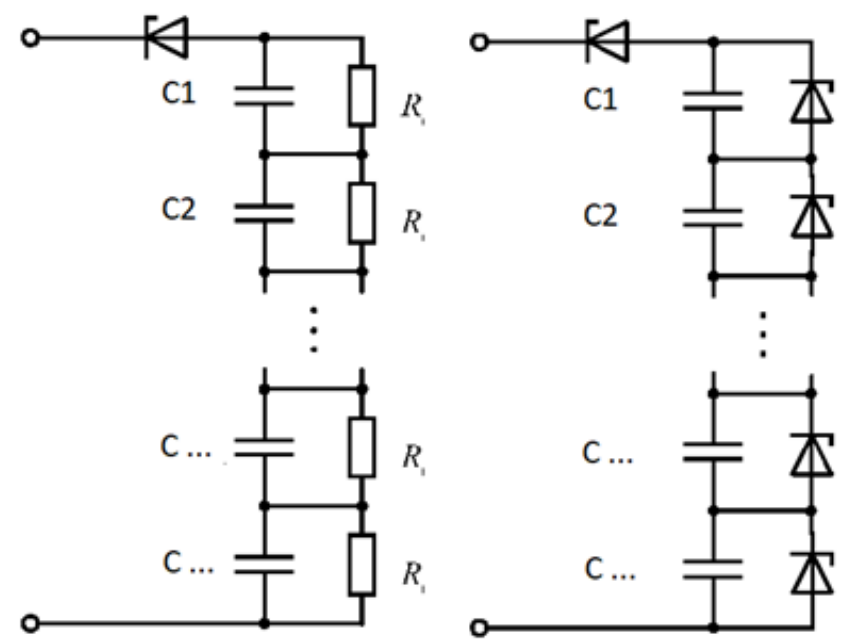

Figure 1 Circuitry scheme of EC module

\section{$4 \quad$ Results and Discussion}

EC modules are based on series connection of EC cells. The modules have a working voltage in a range of 12-24 V according to requirements in applications. Working voltage of $5-15 \mathrm{~V}$ as an example is match with the $12 \mathrm{~V}$ nominal voltage of lead-acid batteries [20].

Figure 2 shows the 15 curves of different galvanostatic variations of EC modules. Each curve consists of two plot lines, i.e., charging as a function of time is in the left part of the curve, meanwhile the right line represents plot of discharging. When the lines are very steep, charging/discharging speed runs fast. In contrary, when the lines are slope, the processes take place slowly. It can be seen from Figure 2 that each profile has a steeper charging line curve than discharging line curve. Thus, the charging process takes place faster than the discharging.

The actual full charging period of EC module lasts for 5-10 minutes, while the discharging process time varies depending on the use of diodes as a resistor component of the current. It can be seen that the use of blocking components causes the discharging process much longer. Respectively, the requiring time for the discharging process ranges from 150,100 and 500 minutes for EC module without or with Zener diode and Schottky diode as blocking components (Figure 2).

Figure 3 shows that the use of blocking components does not significantly increase the charging speed, but more influential to the discharging process. It can be shown from Figure $2(a-c)$ in NA plot that discharging 
line is steeper compared to charging lines in DZ, DS, $\mathrm{R} 100, \mathrm{R} 1 \mathrm{k}$ plots. The discharging process is much longer visible on the use of Schottky diodes as blocking component. Schottky diode then used as a balancing component in EC module. Figure 3 shows its effect of blocking EC module. The use of blocking components inside a module led to stability in the process of charging-discharging EC cells in the module. Figure 3 shows the difference between EC modules with and without the use of a blocking component.

(a)

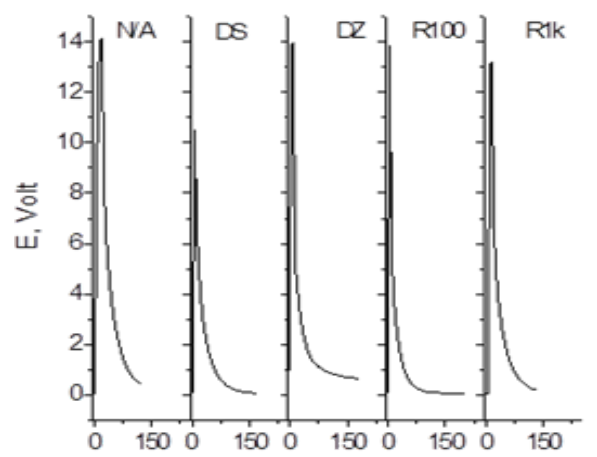

(b)

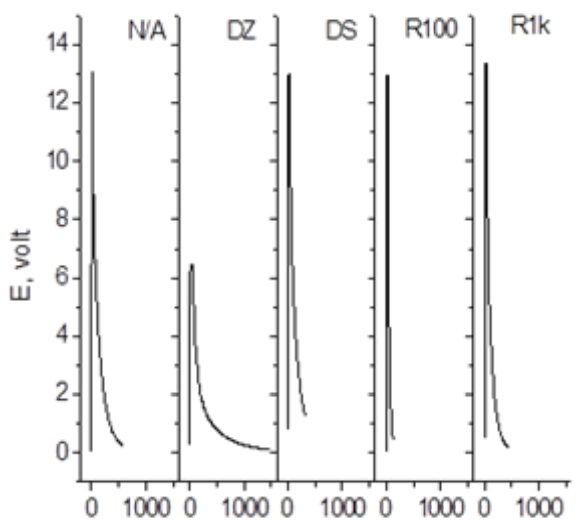

(c)

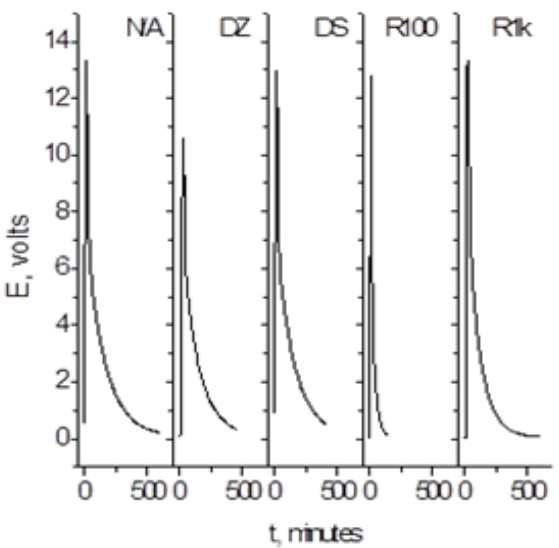

Figure 2 Charging-discharging profile of EC module which equipped with a balancing component $(\mathrm{DZ}=$ Zener Diode, DS $=$ Schottky Diode, $\mathrm{R} 100=$ $100 \mathrm{ohm}$ resistor, $\mathrm{R} 1 \mathrm{k}=1 \mathrm{k}-\mathrm{ohm}$ resistor and $\mathrm{N} / \mathrm{A}=$ no components) and a current blocking component ((a) N/A; (b) with Schottky diodes and (c) with Zener diode).
Continuous balancing causes problems in voltage stability, that in the turn affects lifetime of EC. The EC with low capacitance has lower voltages stability, therefore EC lifetime is low. Overvoltage will not occur in ECs with low capacitance during the discharging process. Inversely, at the charging cycle, the EC with minimum capacitance will subjected an overvoltage. Therefore, the balancing feature can be operated in on-off mode. The balancing can be disabled in the discharging process, otherwise, enabled in the charging. That is why balancing is done when the overvoltage is possible.

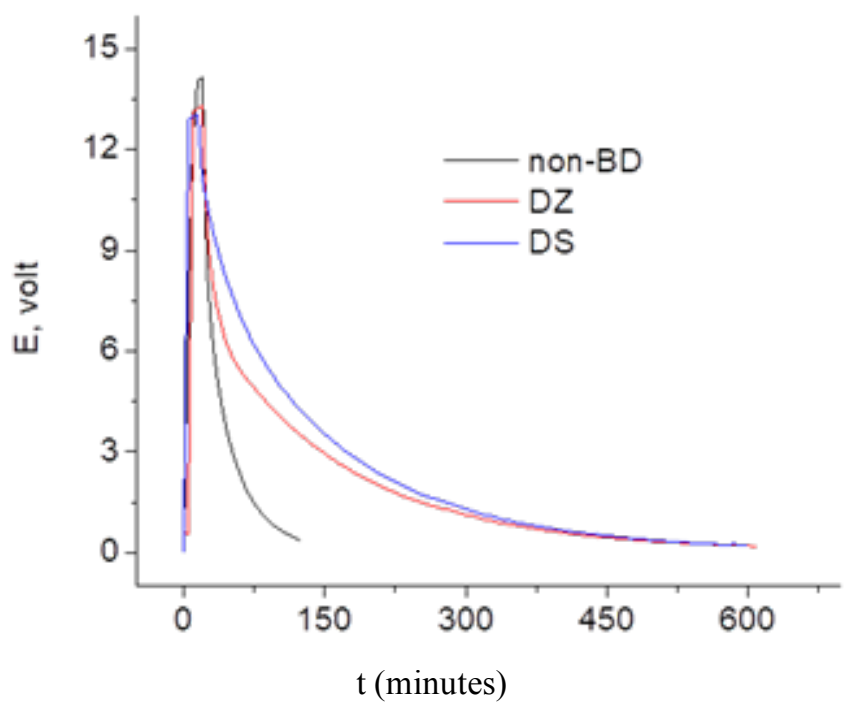

Figure 3 Charging-discharging profile of EC module equipped without balancing component but with current blocking component (non-BD, without blocking diode; DZ, with diode Zener and DS, with Schottky diode).

Figure 4 shows that the use of a balancing component does not have much effect on the process of chargingdischarging process. Only the use of Zener diode (DZ) affect significantly, but it can be said to be a negative effect, which decreases the bias voltage of EC module. If the only consideration is the stability of charging discharging process without considering the other factors, such as the maximum voltage, therefore the components which can be considered as balancer is Schottky diodes and resistors for $1 \mathrm{k}-\mathrm{ohm}$. In other words, these components do not significantly affect the working voltage and the duration of discharge charge cycles on module. Therefore, the use of a blocking and balancing/stabilizing components such as Schottky diodes can be used to observe the stability of the process of charging discharging modules in the long term. 

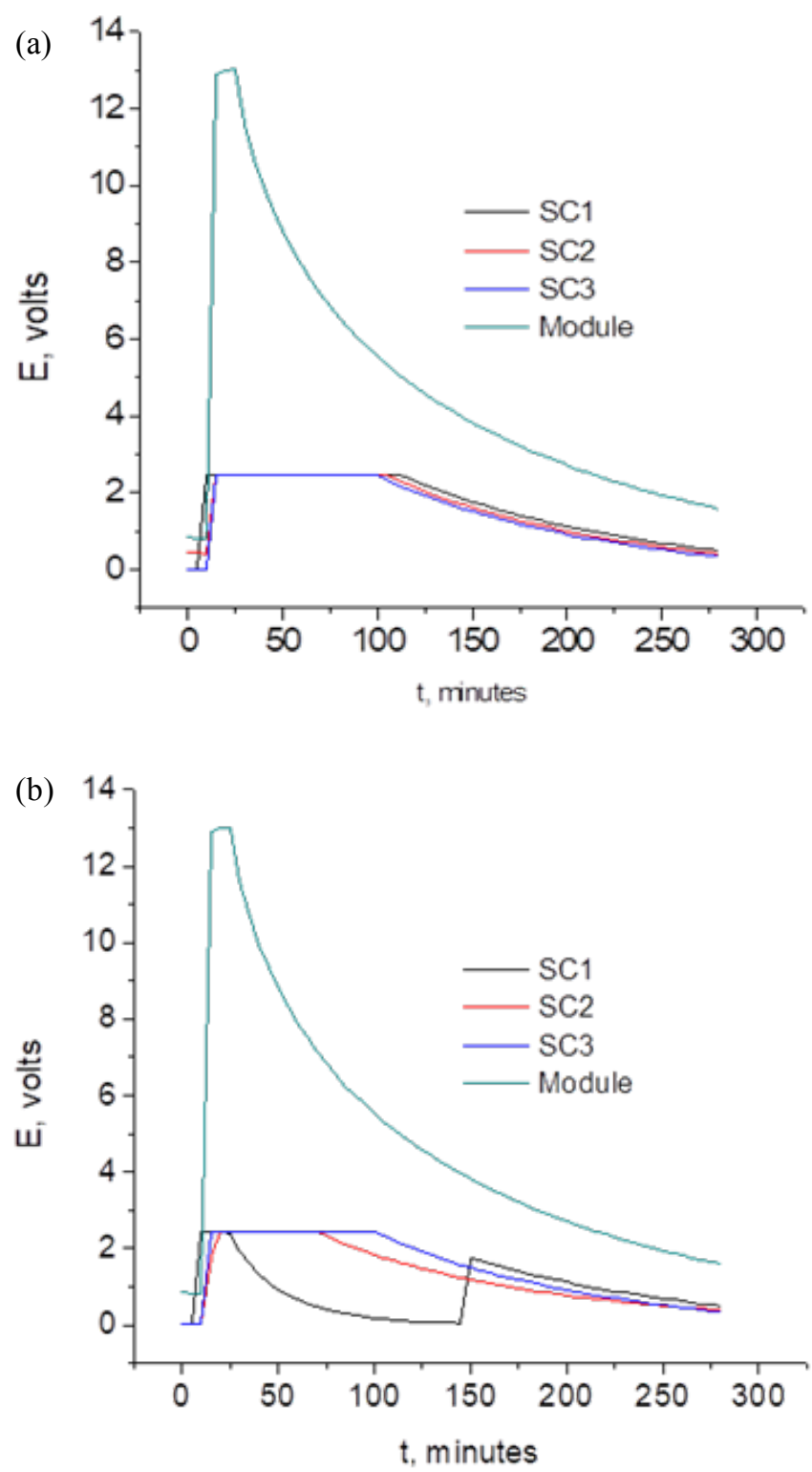

Figure 4 Charging-discharging profile of EC module and each cell: (a) with, and (b) without balancing component (Schottky diode). The measurement was obtained in 1000 cycles.

Diode has an on-off behavior due to its nonlinear current-voltage characteristics. The diode clocks current only in the reverse direction while the reverse voltage is within a limited range and acts as a valve in the electronic and electrical circuit. A P-N junction is the simplest form of the diode which behaves as ideally short circuit when it is in forward-biased mode and behaves as ideally open circuit when it is in the reverse-biased mode. Other way to overcome any potential voltage imbalance is to have resistor connected with EC. The balancing circuit consists of a resistor in series with a controlled switch. This circuit is connected in parallel with each EC. When the switch is on, the balancing current crosses the resistance and drops the voltage of the EC.
Figure 5 is used in assessing stability in charging and discharging processes. It can be seen that the application of Schottky diodes both as blocking and balancing gives stability in charging-discharging process. The balancing system increases the lifetime of ECs as their voltage decrease. The balancing reduces when the overvoltage happens during charging process. Voltage stability decreases when supply voltage devastates balancing resistance in EC during the charging.

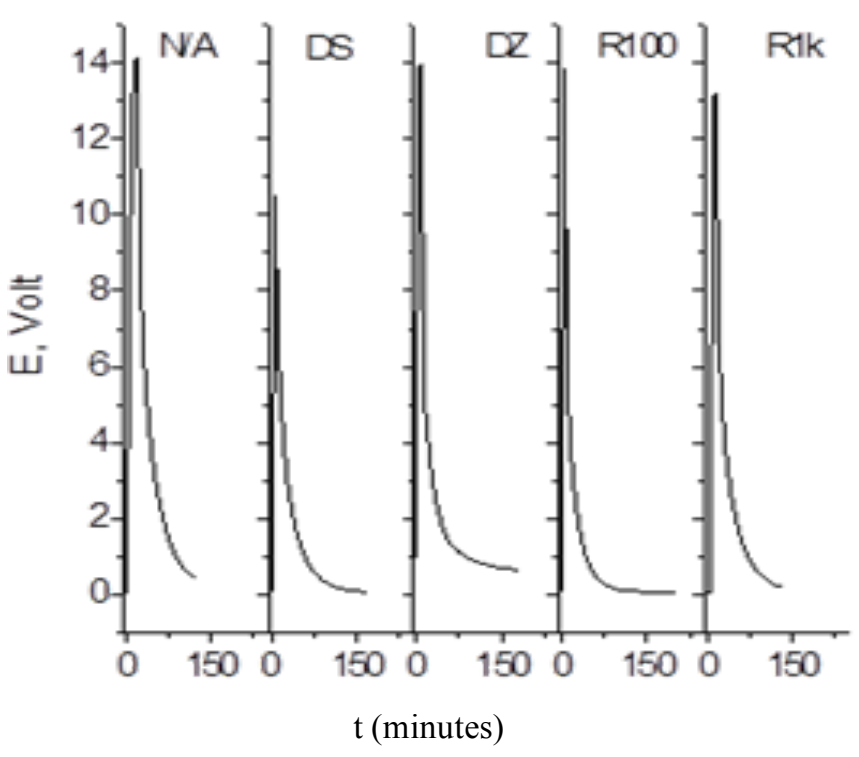

Figure 5 Charging-discharging profile of EC module in 100, 1000, 2000 cycles. The module used Schottky diode both in balancing and current blocking component.

The choice of low capacitance corresponds to the minimum overvoltage limit of all ECs. The control operation, such as on-off mode of stabilizing circuit in EC module. The stabilizing circuit consists of voltage balancing and corresponds to the EC charging discharging process whose overvoltage reduces the voltage - capacitance limit. If overvoltage of the EC reaches the low limit of the voltage - capacitance, then the voltage stability becomes low. Inversely, High limit of the voltage - capacitance in the ECs module make EC more resistant to the overvoltage and the EC achieve greater lifetime [24].

EC has been attracting to be studied as the increasing interest for energy storage applications that provide regulation, contingency and management reserves [25]. The choice of the ideal storage technology to be used depends on a number of factors, among others, the amount of energy or power to be stored, the time for which this stored energy is required to be retained or to be released, spacing and environmental constraints, cost, and the exact location of the network 
on which the storage is required. The improvement in energy storage devices are continuously needed in order to accommodate the increasing sources of voltage instability.

\section{Conclusion}

The use of components does not significantly affect the charging speed, but more influential in the discharging speed. By using Schottky diodes as the blocking component, the discharging process is more visible. Module balancer component using Schottky diodes show the same profile with the condition without it, on the other word, it can be shown that the time and speed of charging process were the same for each balancing component. Charging-discharging profiles have very steep charging curve and much steeper than discharging line. Therefore, the charging process takes place faster than the discharging process. The use of blocking components causes the process to take place much longer in discharging. The use of blocking components inside a module led to stability in the process of charging-discharging EC cells in the module. The use of Zener diode affects significantly, but it can be said to be a negative effect, as it decreases the bias voltage of EC module. the application of Schottky both as blocking and balancing gives stability in charging-discharging process. The balancing system increases the lifetime of ECs by decreasing their voltage.

\section{Acknowledgment}

The authors wish to express deep gratitude to the Lemlit UNSRI for the financial support provided to this work under Research International Collaboration scheme DIPA 605/UN9.3.1/LT/2016 - Universitas Sriwijaya. This paper also partially supported by USAID through Sustainable Higher Education Research Alliances (SHERA) Program - Center for Collaborative (CCR) National Center for Sustainable Transportation Technology (NCSTT) with grant no. IIE00000078-ITB-1.

\section{References}

[1] T. Wei, X. Wei, Y. Gao, and H. Li, "Large scale production of biomass-derived nitrogen-doped porous carbon materials for supercapacitors," Electrochimica Acta, vol. 169, pp. 186-194, Jul. 2015, doi: 10.1016/J.ELECTACTA.2015.04.082.

[2] M. Skrzypkiewicz and S. Obrębowski, "Direct carbon, integrated gasification, and deposited carbon solid oxide fuel cells: a patent-based review of technological status," Journal of Power of Technologies, vol. Vol. 98, pp. 139160, 2018

[3] N. Syarif, "Performance of Biocarbon based Electrodes for Electrochemical Capacitor," Energy Procedia, vol. 52, pp. 18-25, Jan. 2014, doi: 10.1016/J.EGYPRO.2014.07.050.
[4] E. Frackowiak and F. Béguin, "Carbon materials for the electrochemical storage of energy in capacitors," Carbon, vol. 39, no. 6, pp. 937-950, May 2001, doi: 10.1016/S00086223(00)00183-4.

[5] M. Arulepp et al., "The advanced carbide-derived carbon based supercapacitor," Journal of Power Sources, vol. 162, no. 2, pp. 1460-1466, Nov. 2006, doi: 10.1016/J.JPOWSOUR.2006.08.014.

[6] V. V. N. Obreja, "Advance in the assembling and packaging of supercapacitor modules for higher performance," in 2008 2nd Electronics Systemintegration Technology Conference, 2008, pp. 771-774, doi: 10.1109/ESTC.2008.4684448.

[7] B. Xu et al., "Highly mesoporous and high surface area carbon: A high capacitance electrode material for EDLCs with various electrolytes," Electrochemistry Communications, vol. 10, no. 5, pp. 795-797, May 2008, doi: 10.1016/J.ELECOM.2008.02.033.

[8] M. Sun, X. Ma, X. Chen, Y. Sun, X. Cui, and Y. Lin, "A nanocomposite of carbon quantum dots and $\mathrm{TiO}_{2}$ nanotube arrays: enhancing photoelectrochemical and photocatalytic properties," RSC Advances, vol. 4, no. 3, pp. 1120-1127, Nov. 2014, doi: 10.1039/C3RA45474F.

[9] N. C. Das, T. K. Chaki, and D. Khastgir, "Effect of processing parameters, applied pressure and temperature on the electrical resistivity of rubber-based conductive composites," Carbon, vol. 40, no. 6, pp. 807-816, May 2002, doi: 10.1016/S0008-6223(01)00229-9.

[10] X. Luo, J. Wang, M. Dooner, and J. Clarke, "Overview of current development in electrical energy storage technologies and the application potential in power system operation," Applied Energy, vol. 137, pp. 511-536, Jan. 2015, doi: 10.1016/j.apenergy.2014.09.081.

[11] W. van Westering and H. Hellendoorn, "Low voltage power grid congestion reduction using a community battery: Design principles, control and experimental validation," International Journal of Electrical Power \& Energy Systems, vol. 114, p. 105349, Jan. 2020, doi: 10.1016/j.ijepes.2019.06.007.

[12] M. H. Rashid, Ed., Power electronics handbook: devices, circuits, and applications, 2nd ed. Burlington, MA: Academic Press, 2007.

[13] K. Mensah-Darkwa, C. Zequine, P. Kahol, and R. Gupta, "Supercapacitor Energy Storage Device Using Biowastes: A Sustainable Approach to Green Energy," Sustainability, vol. 11, no. 2, p. 414, Jan. 2019, doi: 10.3390/su11020414.

[14] Z. Lu, X. Yu, L. Zhang, X. Meng, L. Wei, and L. Jin, "Experimental investigation on the charge-discharge performance of the commercial lithium-ion batteries," Energy Procedia, vol. 143, pp. 21-26, Dec. 2017, doi: 10.1016/j.egypro.2017.12.642.

[15] R. Tian et al., "Quantifying the factors limiting rate performance in battery electrodes," Nat Commun, vol. 10, no. 1 , p. 1933 , Dec. 2019, doi: 10.1038/s41467-019-097929.

[16] A. M. Saleem, V. Desmaris, and P. Enoksson, "Performance Enhancement of Carbon Nanomaterials for Supercapacitors," Journal of Nanomaterials, vol. 2016, pp. 1-17, 2016, doi: 10.1155/2016/1537269.

[17] Y. Miao, P. Hynan, A. von Jouanne, and A. Yokochi, "Current Li-Ion Battery Technologies in Electric Vehicles and Opportunities for Advancements," Energies, vol. 12, no. 6, p. 1074, Mar. 2019, doi: 10.3390/en12061074.

[18] B. K. Kim, S. Sy, A. Yu, and J. Zhang, "Electrochemical Supercapacitors for Energy Storage and Conversion," in Handbook of Clean Energy Systems, J. Yan, Ed. Chichester, UK: John Wiley \& Sons, Ltd, 2015, pp. 1-25.

[19] P. Kurzweil and M. Chwistek, "Electrochemical stability of organic electrolytes in supercapacitors: Spectroscopy and gas analysis of decomposition products," Journal of Power 
Sources, vol. 176, no. 2, pp. 555-567, Feb. 2008, doi: 10.1016/j.jpowsour.2007.08.070.

[20] M.-Y. Ayad, S. Pierfederici, S. Raël, and B. Davat, "Voltage regulated hybrid DC power source using supercapacitors as energy storage device," Energy Conversion and Management, vol. 48, no. 7, pp. 21962202, Jul. 2007, doi: 10.1016/J.ENCONMAN.2006.12.015.

[21] H. Zhang, Y. Chen, S.-J. Park, and D.-H. Kim, "A Family of Bidirectional DC-DC Converters for Battery Storage System with High Voltage Gain," Energies, vol. 12, no. 7, p. 1289, Apr. 2019, doi: 10.3390/en12071289.

[22] N. Syarif and A. Assaidah, "Multichannel Data Aquisition System for Monitoring Supercapacitor Module And Cells," Telecommunication, Computing, Electronics and Control, vol. 14, no. 4, p. 1307, Dec. 2016, doi: 10.12928/telkomnika.v14i4.3994.

[23] Z. Enhui, Q. Zhiping, and W. Tongzhen, "Research on combination of series and parallel with supercapacitor module," in The 2nd International Symposium on Power Electronics for Distributed Generation Systems, 2010, pp. 685-690, doi: 10.1109/PEDG.2010.5545936.

[24] P. Kreczanik, T. Kovaltchouk, A. Hijazi, P. Venet, and G. Clerc, "Consideration of the ageing in the control of the balancing circuit of supercapacitor," 2010.

[25] P. Nikolaidis and A. Poullikkas, "A comparative review of electrical energy storage systems for better sustainability," Journal of Power Technologies, vol. 97, no. 3, pp. 220-245, 2017. 